\title{
Predictors of post-spinal hypotension in elderly patients; a prospective observational study in the Durban Metropole
}

\author{
ZA Malima, ${ }^{1}$ A Torborg, ${ }^{2}$ L Cronjé,, ${ }^{2}$ BM Biccard ${ }^{3}$
}

\author{
${ }^{1}$ Registrar, Department of Anaesthesiology and Critical Care, University of KwaZulu-Natal, Durban, South Africa \\ ${ }^{2}$ Specialist, Department of Anaesthesiology and Critical Care, University of KwaZulu-Natal, Durban, South Africa \\ ${ }^{3}$ Professor and Second Chair, Department of Anaesthesia and Perioperative Medicine, University of Cape Town, South Africa \\ Corresponding author, email:zolani@gmail.com
}

\begin{abstract}
Aims and objective: To evaluate the pre-spinal risk factors for hypotension associated with spinal anaesthesia in elderly surgical patients.

Summary of background data: Hypotension is associated with morbidity and mortality following noncardiac surgery. Reducing the incidence of hypotension associated with spinal anaesthesia may be associated with improved postoperative outcomes.

Methods: This was a prospective, observational study, using convenience sampling. All patients over 55 years of age scheduled to receive spinal anaesthesia for lower limb surgery were eligible. Exclusion criteria included the need for resuscitation and/or the need for vasopressors prior to anaesthesia and surgery, and patients who declined spinal anaesthesia. The dosage of spinal anaesthesia and the use of intraoperative sedation were left to the attending anaesthesiologist. The primary outcome was hypotension, which was defined as a decrease in the systolic blood pressure by $25 \%$ from the baseline, or a systolic blood pressure below $100 \mathrm{mmHg}$. The following pre-spinal risk factors were assessed in a multivariable logistic regression for their association with spinal hypotension: age, American Society of Anaesthesiologists-Physical September 2019 Status, sex, dose of local anaesthetic, isobaric bupivacaine or bupivacaine with dextrose, baseline blood pressure, baseline heart rate and fluid preloading prior to spinal anaesthesia.

Results: Three hundred and eighty-nine patients were recruited. The primary outcome of spinal hypotension was independently associated with female sex (odds ratio [OR] 1.97, 95\% confidence interval [CI] 1.19-3.28), increasing dosage of bupivacaine (OR $1.14, \mathrm{Cl} 1.01-1.29)$, and the use of isobaric bupivacaine (OR 1.50, Cl 0.95-2.36).

Conclusion: Spinal hypotension was associated with female sex, increasing dosage of bupivacaine, and the use of isobaric bupivacaine.
\end{abstract}

Keywords: spinal anaesthesia, hypotension, elderly, lower limb surgery

\section{Introduction}

Intraoperative hypotension is associated with perioperative morbidity and mortality. ${ }^{1}$ Spinal anaesthesia is commonly performed for lower limb surgery in our setting and may be preferable in this group of patients. ${ }^{2}$ In addition, regional anaesthesia may have some advantages over general anaesthesia with respect to modifying the stress response to surgery, ${ }^{3}$ reducing blood loss at surgery, improving postoperative oxygenation, reducing postoperative pulmonary complications and deep vein thrombosis, and modifying the immune response after surgery. ${ }^{4}$

Awareness of the morbidity associated with perioperative hypotension is increasing. Spinal hypotension could therefore result in morbidity in the elderly surgical population. A better understanding of the risk factors for spinal hypotension may enable us to reduce its incidence. While a number of studies have identified various individual risk factors for spinal hypotension, e.g. dose of local anaesthetic, we are unaware of a study which has examined the association across a combination of these risk factors for spinal hypotension in elderly patients. The objective of this study was to determine the pre-spinal risk factors independently associated with spinal hypotension in elderly surgical patients undergoing lower limb surgery.

\section{Methods}

The study protocol was approved by the University of KwaZuluNatal Biomedical Research Ethics Committee (BREC) BE308/15. Permission from the Department of Health in KwaZulu-Natal to conduct research at Inkosi Albert Luthuli Central Hospital and King Edward VIII Hospital was granted via Health Research and Knowledge Management Department (HRKMD).

This was a prospective, observational study, using convenience sampling. Consent was waived for this study, as it was deemed to be low risk and purely observational. The primary outcome of the study was spinal hypotension, defined as a decrease in the systolic blood pressure by $25 \%$ from the baseline, or a systolic blood pressure below $100 \mathrm{mmHg} .^{5}$

The following risk predictors were included in the analysis: age of the patient, ${ }^{6}$ sex, baricity of the bupivacaine, dosage of bupivacaine (per $0.1 \mathrm{mg}$ increase), ${ }^{7}$ fluid preloading, ${ }^{8,9}$ and baseline heart rate. ${ }^{10}$ Inclusion criteria included surgical patients above the age of 55 years presenting for lower limb surgery, American Society of Anaesthesiologists Physical Status (ASA-PS) class 1-3, who were scheduled to receive spinal anaesthesia. Exclusion criteria were patients who declined regional anaesthesia, required extensive resuscitation prior to the anaesthetic and surgical procedure which we defined 
as fluid bolus of greater than $20 \mathrm{ml} / \mathrm{kg}$, patients requiring vasopressors prior to anaesthesia, and patients with absolute contraindications to neuraxial anaesthesia.

Spinal anaesthesia was conducted in the following manner. Patients received no premedication prior to arriving in the operating theatre. Standard monitoring included non-invasive blood pressure monitoring, three lead electrocardiogram, and pulse oximetry. Venous access was established, using an 18 or 20 gauge (G) cannula. Modified Ringer's lactate solution was the initial fluid used in the operating theatre.

Spinal anaesthesia was conducted with the patients in the seated position using a midline approach. Prior to subarachnoid puncture, sterile techniques were employed, followed by 3-5 $\mathrm{ml}$ of $2 \%$ Lignocaine B.Braun ${ }^{\oplus}$ Solution to the skin. The L3-L4 lumbar space was the first choice for subarachnoid puncture. The standard needles used for spinal anaesthesia were Portex $^{\circledast} 26 \mathrm{G}$ pencil point $90 \mathrm{~mm}$ or a Portex ${ }^{\circledast}$ combined spinal/ epidural minipack with lock pencil point spinal needle (26G/16G respectively). The choices of regional technique, the dosage and type of bupivacaine (isobaric bupivacaine or bupivacaine with dextrose) were left to the discretion of the attending anaesthesiologist. Both $0.5 \%$ bupivacaine spinal with dextrose injection and $0.5 \%$ isobaric bupivacaine spinal injection were acceptable for the spinal block.

Once the block was established, the patient was catheterised and positioned for surgery. The blood pressure was measured at one-minute intervals for the first 15 minutes, and then every five minutes. The level of the block was assessed using loss of cold sensation to ice pack. Hypotension was defined as a decrease in the systolic blood pressure by $25 \%$ or a systolic blood pressure reading below $100 \mathrm{~mm} \mathrm{Hg} .{ }^{5}$ Bradycardia was defined as a heart rate below 50 beats per minute (bpm). The data were captured onto a data collection sheet, from baseline until one hour post administration of spinal anaesthesia at five-minute intervals. The lowest systolic blood pressure and heart rate within a five-minute period were recorded. Sedation choices available were propofol infusion, midazolam 1-3 mg bolus or ketamine 10-30 mg bolus. The choice of vasopressors included phenylephrine $50-100 \mathrm{mcg}$ or ephedrine $5-10 \mathrm{mg}$ as a bolus, which were administered at the discretion of the attending anaesthesiologist.

\section{Statistical analysis}

Based on an expected incidence of spinal hypotension of $15 \%,{ }^{11}$ and in order to ensure greater than ten events per variable, ${ }^{12}$ 350 patients were needed to test five to six potential pre-spinal risk predictors of spinal hypotension in a multivariable logistic regression. An alpha error was set at 0.05 . To maintain an adequate number of events per variable, we intended to enter risk factors with a univariate association of $p<0.1$ for spinal hypotension into multivariate analysis, using binary logistic regression. However, as the incidence of spinal hypotension was higher than predicted, a post hoc decision was taken to enter all the pre-spinal variables into the multivariable regression, as we were still powered for greater than ten events per variable. The odds ratio (OR) for the primary outcome and the $95 \%$ confidence intervals $(\mathrm{Cl})$ are reported. As there is little evidence to suggest whether isobaric bupivacaine or bupivacaine with dextrose should be the indicator variable in a logistic regression model, we selected bupivacaine with dextrose, as the height of the block could be manipulated through patient positioning. Similarly, as there is no guidance on which sex should be the indicator in the model, we selected male sex, as we believed it may be associated with a proportionally smaller dose relative to that in females due to the difference in body size; we believe that males are potentially at lower risk of hypotension than females.

All categorical data were analysed using descriptive statistics and are presented as percentages and $95 \%$ confidence intervals (Cl) where appropriate. Categorical data were analysed using Fisher's Exact Test or Pearson's Chi-square test where appropriate. All continuous data were analysed using descriptive statistics and presented as mean (standard deviation [SD]), as all continuous variables were normally distributed.

\section{Results}

From January to December 2016, a total of 378 patients were recruited into the study. The patient recruitment is shown in Figure 1. Twenty-one (5.6\%) patients had missing data, resulting in 357 (94.4\%) patients included in the final analysis.

The patient characteristics are shown in Table I. On univariate analysis, spinal hypotension was associated with female sex, increasing dosage of bupivacaine, and the use of isobaric bupivacaine. The incidence of spinal hypotension was $56 \%$. The sensory level was tested using a loss of cold sensation to ice pack in 254/357 (71.1\%) patients; of those patients 129 (64.5\%) developed hypotension. Fluid preloading was uncommon prior to spinal anaesthesia with $17(4.8 \%)$ patients receiving a fluid preload (defined as an intravenous fluid bolus of $8 \mathrm{ml} / \mathrm{kg}$ of either modified Ringer's lactate or hydroxyethyl starch [Voluven] prior to commencement of spinal anaesthesia). ${ }^{13}$

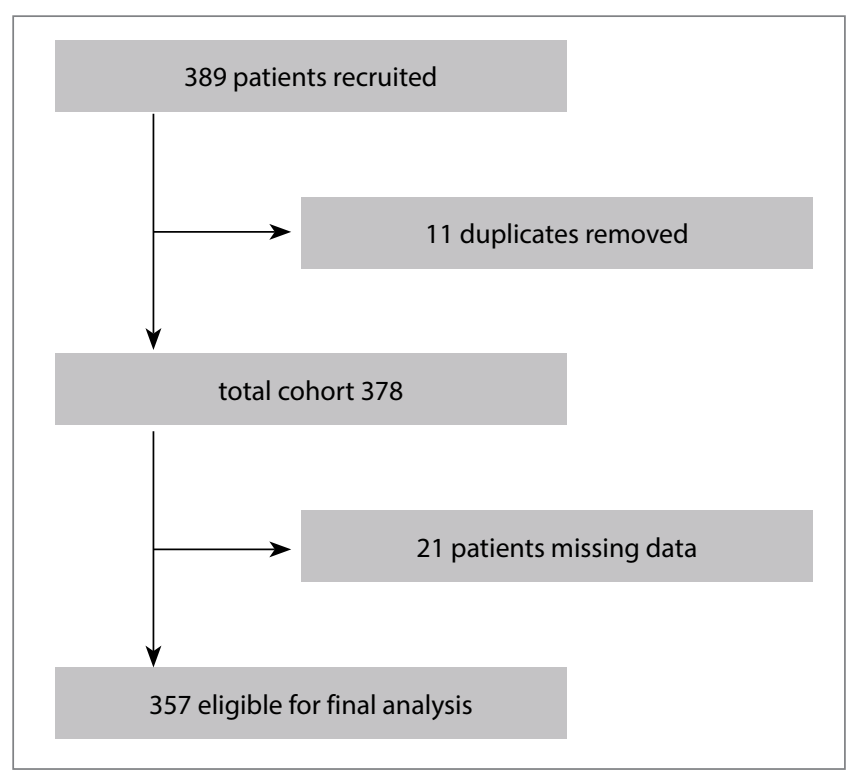

Figure 1. Patient recruitment in the study 
Table I. Patient characteristics and baseline data of the cohort, and the patients with and without spinal hypotension. Data are presented as $\mathrm{n}$ (\%) or mean (SD)

\begin{tabular}{|c|c|c|c|c|}
\hline & All patients $(n=357)$ & Hypotensive $(n=200)$ & Normotensive $(n=157)$ & p-value \\
\hline Age (years) & $67.0(8.3)$ & $67.24(8.1)$ & $66.6(8.5)$ & 0.45 \\
\hline Female sex & $212(59.4 \%)$ & $132(66.0 \%)$ & $80(51.0 \%)$ & $<0.01$ \\
\hline \multicolumn{5}{|l|}{ ASA-PS } \\
\hline $\begin{array}{l}\text { ASA-PS } 1 \\
\text { ASA-PS } 2 \\
\text { ASA-PS } \geq 3\end{array}$ & $\begin{array}{c}15(4.2 \%) \\
147(41.2 \%) \\
195(54.6 \%)\end{array}$ & $\begin{array}{c}9(4.5 \%) \\
98(49.0 \%) \\
87(43.5 \%)\end{array}$ & $\begin{array}{c}6(3.8 \%) \\
49(31.2 \%) \\
97(61.8 \%)\end{array}$ & $\begin{array}{l}1.33 \\
0.60 \\
1.00\end{array}$ \\
\hline \multicolumn{5}{|l|}{ Pre-spinal } \\
\hline HR & $81(17)$ & $82(17)$ & $80(16)$ & 0.22 \\
\hline Baseline HR > 90 & $108(30.3 \%)$ & $64(59.3 \%)$ & $44(40.7 \%)$ & 0.42 \\
\hline Baseline systolic BP & $155(23)$ & $155(22)$ & $155(25)$ & 0.17 \\
\hline Baseline diastolic BP & $84(13)$ & $85(13)$ & $84(13)$ & 0.62 \\
\hline Fluid preload & $17(4.8 \%)$ & $9(4.5 \%)$ & $8(5.1 \%)$ & 0.81 \\
\hline Isobaric bupivacaine & $226(63.3 \%)$ & 137 (68.5\%) & $89(56.7 \%)$ & 0.03 \\
\hline Bupivacaine dose (mg) & $12.2(1.9)$ & $12.4(1.8)$ & $11.9(2.0)$ & 0.02 \\
\hline \multicolumn{5}{|l|}{ Post-spinal } \\
\hline Bradycardia & $103(28.9 \%)$ & $90(45 \%)$ & $13(8.3 \%)$ & $<0.01$ \\
\hline Sensory level tested & $254(71.1 \%)$ & $129(64.5 \%)$ & $125(79.6 \%)$ & $<0.01$ \\
\hline Sedation & $10(2.8 \%)$ & $6(3.0 \%)$ & $4(2.5 \%)$ & 1.00 \\
\hline
\end{tabular}

ASA-PS: American Society of Anaesthesiologists-Physical Status, HR: heart rate, BP: blood pressure, SD: Standard Deviation

Table II. Multivariable analysis of pre-spinal risk factors associated with spinal hypotension in elderly surgical patients

\begin{tabular}{lcc}
\hline & OR (95\% CI) & p-value \\
\hline Age (years) & $1.02(0.99-1.45)$ & 0.19 \\
Female sex & $1.78(1.12-2.81)$ & 0.01 \\
\hline ASA-PS 1 & Reference & \\
\hline ASA-PS 2 & $1.25(0.41-3.79)$ & 0.69 \\
\hline ASA-PS $\geq 3$ & $0.69(0.23-2.07)$ & 0.50 \\
Isobaric bupivacaine & $1.50(0.95-2.36)$ & 0.08 \\
\hline Bupivacaine dose (per 0.1 mg) & $1.14(1.01-1.29)$ & 0.03 \\
\hline Fluid preload & $1.29(0.46-3.63)$ & 0.63 \\
Systolic BP & $1.00(0.99-1.01)$ & 0.99 \\
Diastolic BP & $0.99(0.98-1.01)$ & 0.71 \\
Baseline HR & $1.01(0.99-1.02)$ & 0.17 \\
\hline ASA-PS: Ameican Soiety &
\end{tabular}

ASA-PS: American Society of Anaesthesiologists-Physical Status; BP: blood pressure; HR: heart rate; Cl: Confidence Interval; OR: Odds Ratio

The independent risk factors associated with spinal hypotension are shown in Table II. Female sex, isobaric bupivacaine, and an increasing bupivacaine dose were independently associated with spinal hypotension.

\section{Discussion}

\section{Principal findings}

Hypotension following spinal anaesthesia in elderly patients was common, with an incidence of $56 \%$ in this study. Hypotension was independently associated with female sex, isobaric bupivacaine, and an increasing bupivacaine dose.

\section{Strengths and weaknesses of the study}

The majority of the work in spinal hypotension has been done in the pregnant population. There are few studies which examine risk factors for spinal hypotension in elderly patients. ${ }^{5,10,14}$ The increasing number of elderly patients presenting for surgery warrants an understanding of the risk of spinal hypotension in this patient group. In order to ensure that the results are broadly generalisable, we included both elective and emergency surgical procedures in this study. Furthermore, we powered the study to include most of the plausible pre-spinal risk factors for spinal hypotension.

The potential weaknesses of the study were the following: Firstly, we did not control for patient comorbidities such as hypertension, diabetes and vascular disease. It is possible that these factors may adversely affect the incidence of spinal hypotension, independently of the factors that we have identified. However, we believe that we have adequately controlled for this potential limitation by including the preoperative blood pressures and ASA-PS in the logistic regression model. It is possible that including the preoperative systolic and diastolic blood pressure in the multivariable model is more desirable than controlling for a history of hypertension, particularly as it has been shown in a South African population that over $50 \%$ of adult patients presenting for elective surgery have undiagnosed hypertension. ${ }^{15}$ These patients would be missed based on a history of hypertension, but are likely more accurately represented by inclusion of the preoperative blood pressure, especially as it is the extent of the blood pressure control that is considered important for haemodynamic stability in the perioperative period. ${ }^{16}$ None of these potential pre-spinal haemodynamic risk factors were independently associated with spinal hypotension, and we therefore believe that the impact of a history of hypertension on this model will likely be insignificant. Secondly, although we did not include other specific comorbidities, we have also included the ASA-PS in the 
multivariable logistic regression model, and this too may partly control for other comorbidities. Importantly, spinal hypotension was not associated with an increasing ASA-PS.

Another limitation that we have not controlled for is chronic pharmacological management of the above-mentioned diseases. We would expect some antihypertensive medications to be associated with increased spinal hypotension. ${ }^{17}$

Due to the nature of the pathology of this population group, (i.e. neck of femur fractures, osteoarthritis, diabetic foot sepsis) we were unable to obtain patient weights and heights to calculate body mass index (BMI), and hence could not control for differences in the BMI. This may be a confounder for the identification of female sex as an independent predictor of spinal hypotension.

\section{Interpretation of the study}

As life expectancy increases, more elderly patients will present for various types of surgery. Neuraxial techniques may be considered advantageous in certain elderly patients, for example a patient presenting with chronic pulmonary disease. Studies have shown that regional anaesthesia positively affects some postoperative outcomes, including improved respiratory function, less nausea and vomiting, less pain, and lower incidence of deep vein thrombosis. ${ }^{18}$ However, these benefits need to be weighed against the risk of hypotension, the incidence of which ranges from $8-33 \% .{ }^{11}$ In our study we found an incidence of spinal hypotension of $56 \%$ (95\% Cl 50.9-61.2) which was higher than that reported in the international literature.

Other studies of risk factors associated with spinal hypotension in elderly patients corroborate some of our findings. Pereira et al. showed that patients who were 61 years and older were more prone to develop spinal hypotension. ${ }^{11}$ This is consistent with the high incidence of spinal hypotension in our cohort. Our study also suggests that female sex is associated with a higher risk for developing spinal hypotension, with 132 females of 212 total patients developing spinal hypotension $(66.0 \%, p=0.01)$.

In a study by Olofsson et al., a reduced dose of bupivacaine with dextrose $(7.5 \mathrm{mg}$ ) with addition of sufentanil (5 ug) as a single shot provided adequate spinal block for hip repair in elderly patients with stable haemodynamics. ${ }^{5}$ In our study, we found that a lower dose of bupivacaine was associated with significantly less hypotension. Furthermore, administration of bupivacaine with dextrose was associated with significantly less hypotension, than the use of isobaric bupivacaine.

A baseline heart rate above 90 beats per minute (bpm) was found to be a predictor of spinal hypotension in a study by Meyhoff et al. ${ }^{10}$ However, preoperative HR was not associated with spinal hypotension in our study, and a sensitivity analysis in our study which included a HR $>90 \mathrm{bpm}$ (as opposed to HR as a continuous variable), did not find a HR $>90 \mathrm{bpm}$ to be an independent predictor of spinal hypotension.
Fluid preloading has been associated with less spinal hypotension in elderly patients. ${ }^{13}$ However there is a lack of consensus in the literature as to the type of fluid and the amount of fluid needed for preloading prior to induction of spinal anaesthesia. The literature suggests the use of fluid preloading as being protective against the development of spinal hypotension in elderly patients, and colloids more so than crystalloids. ${ }^{13}$ Xei et al. showed that preloading with a colloid compared to a crystalloid was superior in preventing hypotension at a volume of $8 \mathrm{ml} / \mathrm{kg} .{ }^{13}$ Nakusuji et al. showed that crystalloid intravascular volume expansion prevented a decrease in cardiac output regardless of preloading or co-loading. ${ }^{14}$ Fluid preloading was not associated with a decrease in spinal hypotension, although due to the limited use of fluid preloading in our study, we would suggest that more research is needed to determine if fluid preloading decreases spinal hypotension in elderly surgical patients.

Ten (2.8\%) patients received sedation intraoperatively. As this was administered intraoperatively, it was not entered into the multivariate regression.

\section{Conclusion}

Our study showed that spinal hypotension is common in elderly patients, and it is associated with female sex, bupivacaine dose and isobaric bupivacaine. The attending anaesthesiologist should have a high index of suspicion when any of these risk factors are present, as spinal hypotension may be associated with morbidity in elderly surgical patients.

\section{References}

1. Walsh M, Devereaux PJ, Garg AX, Kurz A, Turan A, Rodseth RN, et al. Relationship between intraoperative mean arterial pressure and clinical outcomes after noncardiac surgery toward an empirical definition of hypotension. Anesthesiology: The Journal of the American Society of Anesthesiologists. 2013;119(3):507-15.

2. Christopherson R, Beattie C, Frank SM, Norris EJ, Meinert CL, Gottlieb SO, et al. Perioperative morbidity in patients randomized to epidural or general anesthesia for lower extremity vascular surgery. Anesthesiology: The Journal of the American Society of Anesthesiologists. 1993;79(3):422-34.

3. Park WY, Thompson JS, Lee KK. Effect of epidural anesthesia and analgesia on perioperative outcome: a randomized, controlled Veterans Affairs cooperative study. Annals of surgery. 2001;234(4):560.

4. Cook P, Davies M, Cronin K, Moran P. A prospective randomised trial comparing spinal anaesthesia using hyperbaric cinchocaine with general anaesthesia for lower limb vascular surgery. Anaesthesia and Intensive Care. 1986;14(4):373-80.

5. Olofsson C, Nygards EB, Bjersten AB, Hessling A. Low-dose bupivacaine with sufentanil prevents hypotension after spinal anesthesia for hip repair in elderly patients. Acta Anaesthesiologica Scandinavica. 2004;48(10):1240-4.

6. Critchley LAH. Hypotension, subarachnoid block and the elderly patient. Anaesthesia. 1996;51(12):1139-43.

7. Martyr JW, Clark MX. Hypotension in elderly patients undergoing spinal anaesthesia for repair of fractured neck of femur. A comparison of two different spinal solutions. Anaesthesia and Intensive Care. 2001;29(5):501-5.

8. Marhofer P, Faryniak B, Oismüller C, Koinig H, Kapral S, Mayer N. Cardiovascular effects of $6 \%$ hetastarch and lactated Ringer's solution during spinal anesthesia. Regional Anesthesia and Pain Medicine. 1999;24(5):399-404.

9. Coe AJ, Revanäs B. Is crystalloid preloading useful in spinal anaesthesia in the elderly? Anaesthesia. 1990;45(3):241-3.

10. Meyhoff CS, Haarmark C, Kanters JK, Rasmussen LS. Is it possible to predict hypotension during onset of spinal anesthesia in elderly patients? Journal of Clinical Anesthesia. 2009;21(1):23-9.

11. Pereira IDF, Grando MM, Vianna PTG, Braz JRC, Castiglia YMM, Vane LA, et al. Retrospective analysis of risk factors and predictors of intraoperative complications in neuraxial blocks at Faculdade de Medicina de Botucatu-UNESP. Brazilian Journal of Anesthesiology. 2011;61(5):568-81. 
12. Peduzzi P, Concato J, Kemper E, Holford TR, Feinstein AR. A simulation study of the number of events per variable in logistic regression analysis. Journal of Clinical Epidemiology. 1996;49(12):1373-9.

13. Xie R, Wang L, Bao H. Crystalloid and colloid preload for maintaining cardiac output in elderly patients undergoing total hip replacement under spinal anesthesia. Journal of Biomedical Research. 2011;25(3):185-90.

14. Nakasuji M, Suh SH, Nomura M, Nakamura M, Imanaka N, Tanaka M, et al. Hypotension from spinal anesthesia in patients aged greater than 80 years is due to a decrease in systemic vascular resistance. Journal of Clinical Anesthesia. 2012;24(3):201-6.

15. Van der Spuy K, Crowther M, Nejthardt M, Roodt F, Davids J, Roos J, et al. A multicentre, cross-sectional study investigating the prevalence of hypertensive disease in patients presenting for elective surgery in the Western Cape Province, South Africa. South African Medical Journal. 2018;108(7).

16. Hartle A, McCormack T, Carlisle J, Anderson S, Pichel A, Beckett N, et al. The measurement of adult blood pressure and management of hypertension before elective surgery: Joint Guidelines from the Association of Anaesthetists of Great Britain and Ireland and the British Hypertension Society. Anaesthesia. 2016;71(3):326-37.

17. Roshanov PS, Sheth T, Duceppe E, Tandon V, Bessissow A, Chan MT, et al. Relationship between perioperative hypotension and perioperative cardiovascular events in patients with coronary artery disease undergoing major noncardiac surgery. Anesthesiology: The Journal of the American Society of Anesthesiologists. 2019;130(5):756-66.

18. Bajaj P. Regional anaesthesia versus general anaesthesia: Is there an impact on outcome after major surgery? Indian Journal of Anaesthesia. 2007;51(2):153. 